\title{
Express Paper
}

\section{Breast MR Image Fusion by Deformable Implicit Polynomial (DIP)}

\author{
Bo Zheng $^{1, \text { a) }} \quad$ Ryo Ishikawa $^{3, b)}$ Jun Takamatsu $^{1, c)}$ Katsushi Ikeuchi $^{1, d}$ ) \\ TakaAki Endo ${ }^{3, \mathrm{e})}$ Kiyohide $\mathrm{Sato}^{3, \mathrm{f})}$ Takayuki Ueno ${ }^{4, \mathrm{~g})}$ Tomoharu Sugie ${ }^{5, \mathrm{~h})}$ \\ Masakazu Tol $^{4, \mathrm{i})}$ Shotaro Kanao ${ }^{6, \mathrm{j})} \mathrm{KaOri}$ Togashi $^{6, \mathrm{k})}$ \\ Received: March 11, 2013, Accepted: April 24, 2013, Released: July 29, 2013
}

\begin{abstract}
MR Image fusion is desired in various image-guide breast surgeries. However it often suffers from the difficulty on dealing with large deformation of breast. This paper presents a novel method for efficiently modeling and inferring the physical parameters, including gravity, Young's modulus, Poisson's ratio, etc, which are important elements for handling the biomechanical deformations of breast with finite element model. Our method consists of two major steps: 1) deformation modeling and 2) non-rigid registration. The former builds a deformable implicit polynomial (DIP) model to encode the physical parameters according to deformation. The latter fast registers the prior DIP to the online breast image such that the image fusion can be achieved. Experimental results demonstrate the good performance of our method.
\end{abstract}

Keywords: breast MR image, non-rigid image registration, implicit polynomial

\section{Introduction}

Magnetic Resonance (MR) images of breast captured in different poses of patients pre- or operatively are widely used in clinics. To the end of breast diagnosis or image guidance of breast surgery, these images are often desired to fuse together to provide rich information. However, the appearance of breast images vary in large scale due to deformation of soft body. For example, Fig. 1 (a) and (b) show two MRI images taken from one patient by prone and supine postures respectively, but they look very different on shape boundary. Furthermore such supine MRI images are commonly with low image quality that they are difficult to be used for detecting breast malignancies (compare the manually labeled circle markers in Fig. 1 (a) and (b)).

To complete the image fusion task, an effective non-rigid image registration method is required. For example, the image in

IIS, U-Tokyo, Meguro, Tokyo 153-8505, Japan

NAIST, Ikoma, Nara 630-0192, Japan

Medical Imaging Project, Canon Inc. Ohta, Tokyo 146-8501, Japan

Department of Breast Surgery, Kyoto University, Kyoto 606-8501, Japan

Department of Surgery, Kansai Medical University, Moriguchi, Osaka 570-8506, Japan

6 Department of Diagnostic Radiology, Kyoto University, Kyoto 6068501, Japan

a) zheng@cvl.iis.u-tokyo.ac.jp

b) ishikawa.ryo@canon.co.jp

j-taka@is.naist.jp

ki@cvl.iis.u-tokyo.ac.jp

endo.takaaki@canon.co.jp

sato.kiyohide@canon.co.jp

takayuki@kuhp.kyoto-u.ac.jp

sugie@kuhp.kyoto-u.ac.jp

toi@kuhp.kyoto-u.ac.jp

kanaos@kuhp.kyoto-u.ac.jp

ktogashi@kuhp.kyoto-u.ac.jp

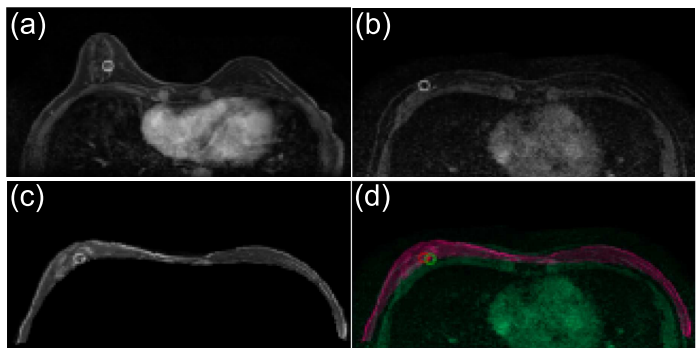

Fig. 1 Fusion of MR images captured by different poses of patient: (a) prone and (b) supine. (c) deformed image of (a). (d) image fusion of (b) and (c).

Fig. 1 (a) can be first deformed to the image shown in (c), and then (c) can be fused with (b) as the result shown in (d).

Plenty of image-based methods have been developed, e.g., one of the most classical mothers is proposed by Rueckert et al. in Ref. [7], where breast motion is defined by transformation of global affine and local free-form deformation (FFD), and mutual information is used as voxel-based similarity measurement. More comprehensive survey is given by Guo et al. in Ref. [3], e.g., intensity-based and feature-based methods are common types for breast image registration which warp image pairs by maximizing the similarities defined on intensities or image features. However these approaches do not make the image deformation faith to the physical reality of breast deformation, so that the place of malignancies cannot be guaranteed to match together. Especially failure cases often happen when dealing with the large deformation or there are few overlaps of consistent image intensities or features.

Physics-based methods guarantee the truth of physical deformation, e.g., Carter et al. [2], [5], [6] consider the factor of grav- 


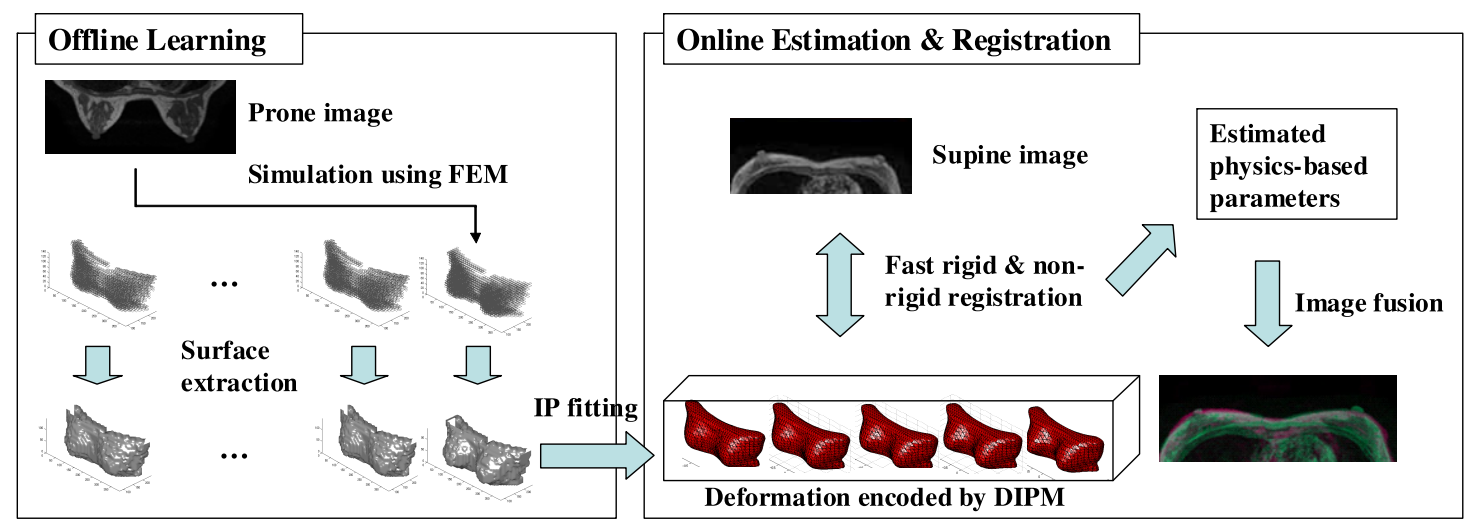

Fig. 2 Method overview.

ity of breast and successfully achieve the registration using FEM (Finite Element Method). However, in the case for estimating physics-based parameters, it is too time-consuming, since large number of hypothetical simulations generated by FEM are necessary to be explored. To speed up FEM model, Hu et al. [4] adopted statistical motion model (SMM) representing the FEMsimulated models for prostate gland image registration. However, unlike FEM, SMM misses the physical parameters, such as gravity, Young's modulus and Poisson's ratio, which would be very helpful for physically and volumetrically simulating the deformation of breast. Furthermore these patient-specific physical parameters can be reused for diagnosis with simulation despite the boundary conditions changed in future.

We propose a novel image fusion technique for breast MRI image by fast estimating the physical parameters that can drive a physical deformation (e.g., Fig. 1 (c)) and fuse the deformed image to operative image (e.g., Fig. 1 (d)). As shown in Fig. 2, our method consists of two processes: offline modeling and online estimation and registration. In off-line modeling, we model the physical deformation of breast by a compact and continuous model termed as: deformable implicit polynomial (DIP) model, which encodes physical parameters previously simulated by FEM from a preoperative image (e.g., prone image). In online image fusion, a high-speed registration between prior DIP and an operative breast image (e.g., supine image) is performed. As result, the appropriate physics-based parameters can be estimated out and thus serves for physics-based image fusion.

Over traditional methods, three major contributions of this paper are: 1) a new efficient model is proposed for representing deformation; 2) a modeling technique is proposed for obtaining compact DIP models; 3) a solution for fast non-rigid registration using DIP is proposed.

\section{Offline Modeling}

As shown in Fig. 2 left part, the first step of our approach, offline learning, is to model the deformation physically simulated by FEM, after the preoperative prone MR image data is captured. Then a compact DIP is build to fit the surface data extracted from FEM models.

\subsection{FEM Simulation}

Given a patient-specific prone breast image, we first build a large number of 3D deformation data by physical simulation using FEM [2]. Each data is calculated according to FEM simulation under a hypothesis of parameter settings, e.g., setting gravity, Young's Modulus and Poisson's ratio with certain values. Secondly only the 3D points on surface of each FEM model are used for modeling. The data set can be viewed as $N k$-dimension data points $\left\{\mathbf{x}_{i}\right\}_{1}^{N}$, each point $\mathbf{x}_{i}$ is a $k$-dimension vector combining a $3 \mathrm{D}$ point and its corresponding physical parameters, e.g., $\mathbf{x}_{i}=(x y z g p)$, where $(x, y, z)$ is the coordinate of 3D point, and $g$ and $p$ are Young's Modulus and Poisson's ration respectively.

\subsection{Deformable Implicit Polynomial Model}

Then our DIP representing dataset $\left\{\mathbf{x}_{i}\right\}_{1}^{N}$ can be defined in a multivariate implicit polynomial form. For example, a 5-variable DIP of degree $n$ is denoted by:

$$
\begin{aligned}
f_{n}(\mathbf{x}) & =\sum_{0 \leq i, j, k, l, m ; i+j+k+l+m \leq n} a_{i j k l m} x^{i} y^{j} z^{k} g^{l} p^{m} \\
& =\sum_{c=1}^{K} a_{c} m_{c}(\mathbf{x}),
\end{aligned}
$$

where $a_{i j k l m}$ is the polynomial coefficient corresponding to monomial $x^{i} y^{j} z^{k} g^{l} p^{m}$. This linear representation can be reordered by index $c$ in lexicographical order. Then $m_{c}$ is the $c$-th monomial of polynomial.

\subsection{DIP Fitting}

Fitting a DIP to dataset $\left\{\mathbf{x}_{i}\right\}_{1}^{N}$ can be viewed as to find an appropriate polynomial $f(\mathbf{x})$, of which the zero set $\left\{\mathbf{x} \mid f_{n}(\mathbf{x})=0\right\}$ can "best" represent the data set. This problem can be solved by linear least-squares optimization as

$$
M^{\mathrm{T}} M \mathbf{a}=M^{\mathrm{T}} \mathbf{b},
$$

where $M$ is the matrix of monomial whose $c$-th column $\mathbf{m}_{c}$ is $\left(m_{c}\left(x_{1}\right), m_{c}\left(x_{2}\right), \ldots, m_{c}\left(x_{N}\right)\right)^{\mathrm{T}}$; $\mathbf{a}$ is unknown coefficient vector; and $\mathbf{b}$ is a non-zero vector that is obtained by numerical stabilization proposed by Blane et al. [1].

Then we adopt QR decomposition proposed by Zheng et al. [9] to make: $M=Q R$, where $Q$ is an orthonormal matrix and $R$ is an upper triangular matrix. Then Eq. (2) can be derived to be an upper-triangular linear system of equations as:

$$
R^{\mathrm{T}} Q^{\mathrm{T}} Q R \mathbf{a}=R^{\mathrm{T}} Q^{\mathrm{T}} \mathbf{b} \rightarrow R \mathbf{a}=\underbrace{Q^{\mathrm{T}} \mathbf{b}}_{\widetilde{\mathbf{b}}} .
$$


Note, it namely means that the linear basis (the columns of M) $\left\{\mathbf{m}_{1}, \mathbf{m}_{2}, \cdots, \mathbf{m}_{c}\right\}$ are orthonormalized to orthonormal basis $\left\{\mathbf{q}_{1}, \mathbf{q}_{2}, \cdots, \mathbf{q}_{c}\right\}$ (the columns of $Q$ ).

In this paper we employ the incremental fitting scheme also proposed by Zheng et al., [9]. That is, Eq. (2) will be solved incrementally by assuming the number of $M$ 's columns $\left\{\mathbf{m}_{1}, \mathbf{m}_{2}, \cdots, \mathbf{m}_{c}\right\}$ increases one by one at each step. By QR decomposition as carried out in Eq. (3), equivalently Eq. (2) will be solved incrementally as:

$$
R_{1} \mathbf{a}_{1}=\widetilde{\mathbf{b}}_{1}, \ldots, R_{c} \mathbf{a}_{c}=\widetilde{\mathbf{b}}_{c},(c=1,2, \ldots)
$$

where the dimension of the upper-triangular linear system at each step is larger than the precede step by one. This iteration will be terminated, once the desired fitting accuracy is satisfied.

\subsection{Compact Fitting}

In our previous study, implicit polynomial has the good property of fewer coefficients than other function based representation, such as splines, (see Ref. [9]). While modeling a high dimensional DIP however, it still faces the challenge that the coefficient space should be huge and thus suffers from high computational cost. For example, if the variable $\mathbf{x}$ of Eq. (1) is a 7-D vector for a polynomial of 8 th degree, there needs 6,435 coefficients need to be solved, which is a big challenge for a modern computer.

In this paper, we propose a novel method for pursuing a compact representation (with sparse coefficients), even if the input is a high-dimension fitting dataset. To this end, we modify the incremental scheme in Eq. (4) by removing the columns in $\mathbf{m}_{c}$ companied with coefficients $a_{c}$ that have little contribution on fitting. That is, we check fitting error (defined later) at each incremental step in Eq. (4), and then do not take the coefficients account into calculation if the fitting error varies too little. Our method is based on two following theorems:

Theorem 1 At the c-th step of incremental scheme Eq. (4), if the last (c-th) diagonal element of matrix $R_{i}$ satisfies: $r_{c}=0$, then matrix M in Eq. (2) is singular.

proof: Suppose $\left\{\mathbf{m}_{1}, \mathbf{m}_{2}, \cdots, \mathbf{m}_{c}\right\}$ are the columns of $M$. According to the orthogonalization theory (also see Gram-Schmidt process in Ref. [9]), $r_{c}=\left\|\mathbf{m}_{c}-\sum_{j=1}^{c-1} r_{j c} \mathbf{q}_{j}\right\|$, where $\left\{\mathbf{q}_{1}, \mathbf{q}_{2}, \cdots, \mathbf{q}_{c-1}\right\}$ are the orthonormalized vectors of $\left\{\mathbf{m}_{1}, \mathbf{m}_{2}, \cdots, \mathbf{m}_{c-1}\right\}$. Thus if $r_{c}=0$, then $\mathbf{m}_{c}=\sum_{j=1}^{c-1} r_{j c} \mathbf{q}_{j}$, i.e., $\mathbf{m}_{c}$ can be linearly represented by vectors $\left\{\mathbf{q}_{1}, \mathbf{q}_{2}, \cdots, \mathbf{q}_{c-1}\right\}$, and equivalently by $\left\{\mathbf{m}_{1}, \mathbf{m}_{2}, \cdots, \mathbf{m}_{c-1}\right\}$. $\square$

Theorem 2 At the c-th step of incremental scheme, if the last entry of $\widetilde{\mathbf{b}}_{c}$ satisfies: $\widetilde{b}_{c}=0$, then the current step has no contribution for reducing least squared errors.

proof: Suppose fitting error is defined as Least Squares (LS) error at the $c$-th step: $E_{c}=\left\|M_{c} \mathbf{a}_{c}-\mathbf{b}\right\|^{2}$, then by substituting $M_{c}=Q_{c} R_{c}$, we can obtain:

$$
\begin{aligned}
E_{c} & =\mathbf{a}_{c}^{\mathrm{T}} R_{c}^{\mathrm{T}} Q_{c}^{\mathrm{T}} Q_{c} R_{c} \mathbf{a}_{c}-2 \mathbf{a}_{c}^{\mathrm{T}} R_{c}^{\mathrm{T}} Q_{c}^{\mathrm{T}} \mathbf{b}+\mathbf{b}^{\mathrm{T}} \mathbf{b} \\
& =-\left\|\widetilde{\mathbf{b}_{c}}\right\|^{2}+\|\mathbf{b}\|^{2} .
\end{aligned}
$$

Then if $\widetilde{b}_{c}=0$, it has no effect on $\left\|\widetilde{\mathbf{b}}_{c}\right\|$ and thus has no contribution to $E_{i}$ compared to $E_{i-1}$. $\square$
Theorem 1 implies the fact that once $r_{c}=0$, the column $\mathbf{m}_{c}$ of $M$ will be collinear to the preceding columns $\left\{\mathbf{m}_{1}, \mathbf{m}_{2}, \cdots, \mathbf{m}_{c-1}\right\}$ which is thus meaningless if orthogonalized. Theorem 2 implies the fact that once the last element of $\widetilde{\mathbf{b}}_{c}, \widetilde{b}_{c}$, is close to zero, there is little contribution for reducing the LS errors and thus the current incremental step has little contribution to overall fitting.

Based on these two observations, we define a criterion for detecting the trivial parameters as: $r_{c} \times b_{c}^{2}<T$, suppose $T$ is a certain threshold. That is, if this criterion is satisfied, column $\mathbf{m}_{i}$ will be discarded and the dimension of Eq. (4) will not grow up. As result, this elimination makes the upper-triangular linear system in Eq. (4) always compact, such that we can obtain a DIP with compact coefficient set.

\section{Online Estimation and Registration}

As illustrated in Fig. 2 right part, given a DIP prior and the input patient-specific supine image, the image fusion will be achieved by non-rigid registration between DIP and 3D point set $\left\{\mathbf{b}_{i}\right\}_{1}^{N}$ that should be boundary points of breast (of the supine image). To this end, we propose to perform rigid and non-rigid registration alternatively.

We formulate the registration problem as an energy minimization problem:

$$
\left\{\mathbf{p}^{\text {rigid }}, \mathbf{p}^{\text {physic }}\right\}=\arg \min _{\mathbf{p}_{1}, \mathbf{p}_{2}} E\left(\mathbf{p}_{1}, \mathbf{p}_{2}\right) .
$$

where $\mathbf{p}_{1} \in \mathbb{R}^{6}$ consists of six parameters for rigid transformation, and $\mathbf{p}_{2}$ consists of the physics-based parameters for nonrigid transformation.

\subsection{Rigid Registration}

Rigid registration starts by fixing $\mathbf{p}_{2}$ with constant values. To minimize Eq. (6), point set $\left\{\mathbf{b}_{i}\right\}$ will be rigidly driven to DIP in defined algebraic distance field in a region $\Omega$ as:

$$
\left\{\mathbf{p}^{\text {rigid }}\right\}=\arg \min _{\mathbf{p}_{1}} \int_{\Omega} \operatorname{dist}\left(T\left(\mathbf{p}_{1}, \mathbf{b}_{i}\right), f_{n}\right) d \Omega,
$$

where $T\left(\mathbf{p}_{1}, \mathbf{b}_{i}\right)$ returns the rigidly transformed points of $\mathbf{b}_{i}$ according to parameter $\mathbf{p}_{1}$; function $\operatorname{dist}(\cdot)$ means the distance from the point $\mathbf{b}_{i}$ to the zero set of $f_{n}$ defined as (see Ref. [8]):

$$
\operatorname{dist}\left(\mathbf{b}_{i}, f_{n}\right)=\frac{f_{n}\left(\mathbf{b}_{i}\right)^{2}}{\left\|\nabla_{p_{1}} f_{n}\left(\mathbf{b}_{i}\right)\right\|^{2}}, \forall \mathbf{x} \in \Omega
$$

where $\nabla_{p_{1}} f_{n}(\mathbf{x})=\left(\frac{\partial f}{\partial p_{1}^{1}}, \frac{\partial f}{\partial p_{1}^{2}}, \ldots, \frac{\partial f}{\partial p_{1}^{n}}\right)$ (suppose $\mathbf{p}_{1}=$ $\left.\left(p_{1}^{1}, p_{1}^{2}, \ldots, p_{1}^{n}\right)\right)$. To minimize Eq. (7), we employ the method proposed by Zheng et al. [8], which registers a rigid IP model to discrete point set. Then rigid registration will be replaced by non-rigid registration once energy function in Eq. (7) converges slowly.

\subsection{Non-rigid Registration}

Non-rigid registration is performed under fixing the rigid transformation parameter $\mathbf{p}_{1}$. Then DIP non-rigidly deforms to fit the point set $\left\{\mathbf{b}_{i}\right\}_{1}^{N}$ by minimizing energy.

$$
\left\{\mathbf{p}^{\text {physic }}\right\}=\arg \min _{\mathbf{p}_{2}} \int_{\Omega} \operatorname{dist}\left(\mathbf{b}_{i}, f\left(\mathbf{p}_{2}\right)\right) d \Omega,
$$


where distance function is similarly defined as: $\operatorname{dist}\left(\mathbf{b}_{i}, f_{n}\right)=$ $\frac{f_{n}^{2}\left(\mathbf{b}_{i}\right)}{\left\|\nabla_{p_{2}} f_{n}\left(\mathbf{b}_{i}\right)\right\|^{2}}$, where $\nabla_{p_{2}} f=\left(\frac{\partial f}{\partial p_{2}^{1}}, \frac{\partial f}{\partial p_{2}^{2}}, \ldots, \frac{\partial f}{\partial p_{2}^{n}}\right)$ (suppose $\mathbf{p}_{2}=$ $\left.\left(p_{2}^{1}, p_{2}^{2}, \ldots, p_{2}^{n}\right)\right)$. The minimization of Eq. (9) can be viewed as a general nonlinear minimization problem and we use the standard Newton method for solving it.

\section{Experimental Results}

Phantom and clinical data are employed for evaluation.

\subsection{Breast Phantom Data}

Data acquisition and modeling The first validation is based on prone-supine MR image registration, using breast phantom CIRS-051 (http://www.cirsinc.com/) made of water-based polymer shown in Fig. 3(a). First the prone MR image is taken by setting the MR shooting range as $300 \times 300 \times 144 \mathrm{~mm}^{3}$ (Fig. 3 (b)). We build the FEM model using the mesh of 9,422 hexahedrons with size of $5 \times 5 \times 5 \mathrm{~mm}^{3}$ for each hexahedron, as shown in Fig. 3 (c). Then we perform 36 simulations of FEM by setting the range of Young's Modulus and Poisson's Ratio as: $\{2000,2400,2800,3200,3600,4000\}$ [pa] and $\{0.0,0.1,0.2,0.3,0.4,0.5\}$ respectively, since Young's Modulus and Poisson's Ratio are two of most important parameters for biomechanical deformation of breast. We also assume the density of the phantom is $1 \mathrm{~g} / \mathrm{cm}^{3}$.

Table 1 shows the modeling result of our method compared to the method [9]. It shows that 1) our method obtained the com-

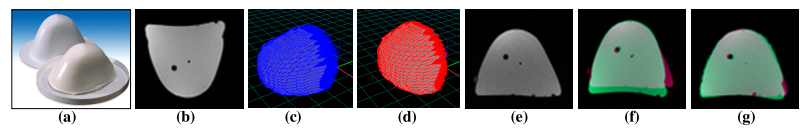

Fig. 3 (a) CIRS-051 phantom; (b) prone MR image of (a); (c) and (d) simulated data using FEM (e) Supine image; (f) Rigid registration result between $(\mathrm{b})$ and $(\mathrm{e}) ;(\mathrm{g})$ our registration result. pact DIP with 102 coefficients without losing learning accuracy, compared to the result of method \#1 using full 462 coefficients required for 6-degree IP; 2) however the result of method \#2, with same number of coefficients to ours, lost too much accuracy to fail for registration.

Registration and estimation After Obtained the DIP, first we test the registration using a synthetic data as the target image with Young's Modulus and Poisson's Ratio of $\{2000$ pa, 0.1\}. Then the DIP is set with same random positions and physics-based parameters of $\{4000 \mathrm{pa}, 0.5\}$. Table 1 shows the estimation result. Note method \#2 failed to perform this registration. Figure 4 illustrates the registration process. Figure $3(\mathrm{e})-(\mathrm{g})$ also shows the prone-supine image registration result using our learned DIP.

\subsection{Clinical Data}

The second evaluation is based on the prone-supine breast clinical data captured from patients, see Fig. 5. The MR acquisition ranges are set as: $350 \times 250 \times 215 \mathrm{~mm}^{3}$ and $380 \times 300 \times 125 \mathrm{~mm}^{3}$ (slice interval is $1.25 \mathrm{~mm}$ ) for prone and supine images respectively. For evaluating the registration accuracy, we first manually marked the locations of breast lesion on both prone and supine images, and assume that for images of same patient the marked locations are completely coincided in the registered images. Then we check the error of the marked lesion location after registration.

To perform registration, we build 24 FEM simulation data
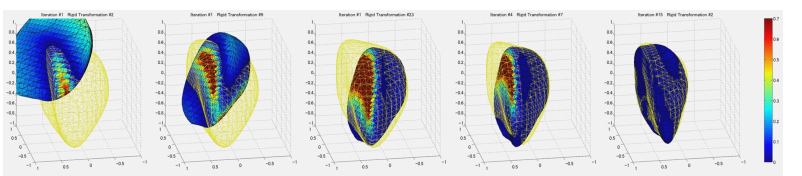

Fig. 4 Registration process consisting of rigid transformation of target (surface model) and non-rigid transformation of DIP (mesh model). Color illustrates the local distance to yellow mesh.

Table 1 Comparison between the method of [9] and our method. Columns from left to right: if coefficient reduction used, number of coefficients, learning error (MSE), and the resulted Young's Modulus $\&$ Poisson's Ratio, registration error (MSE) and running time.

\begin{tabular}{r|l|l|l|l|l|l}
\hline \hline & \multicolumn{3}{|c|}{ Off-line learning } & \multicolumn{3}{c}{ On-line estimation \& registration } \\
\cline { 2 - 7 } & with CR & \# of Coef. & learn. E. $(\mathrm{mm})$ & Y. (pa) \& P. & reg. E. (mm) & time(s) \\
\hline method \# 1 [9] & NO & 462 & 0.715 & $(2003,0.092)$ & 0.903 & 10.27 \\
\hline method \# 2 [9] & NO & 102 & 13.7 & NG & NG & NG \\
\hline our method & YES & 102 & 0.722 & $(2006,0.089)$ & 0.904 & 2.31 \\
\hline
\end{tabular}

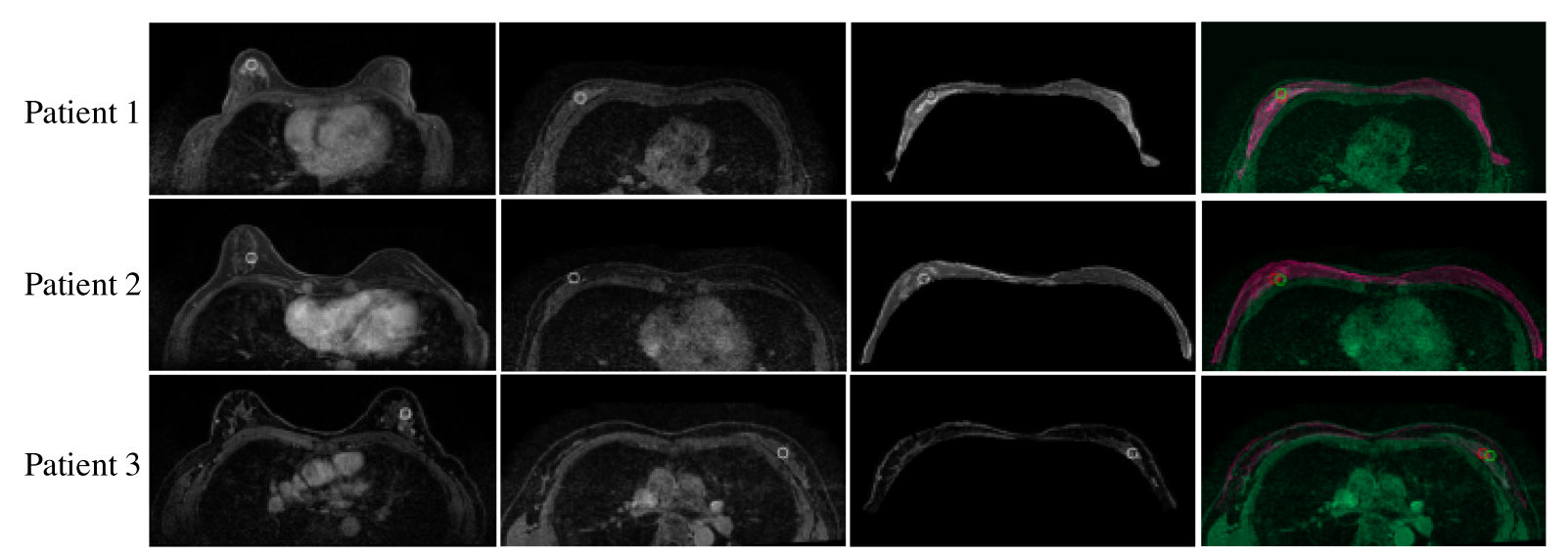

Fig. 5 (a) Clinical prone MR images and (b) supine MR images; (c) Simulated supine images from (a) using estimated parameters; (d) Image fusion of (b) and (c). 
Table 2 Evaluation of registration error between lesion position in prone and in supine.

\begin{tabular}{c|c|c|c|c}
\hline \hline \# Patient & \# Coef. & Young. [Pa] & Poisson's R. & Reg. Err. [mm] \\
\hline 1 & 126 & 426 & 0.33 & 4.341 \\
2 & 120 & 475 & 0.38 & 10.289 \\
3 & 137 & 447 & 0.12 & 10.115 \\
\hline
\end{tabular}

for each clinical case by setting range of Young's Modulus and Poisson's Ratio as: $\{300,400,500,1000\}[\mathrm{pa}]$ and $\{0.0,0.1,0.2,0.3,0.4,0.5\}$ respectively, which are then modeled by an 8-degree DIP. As results, for each case, the number of selected coefficients of learned DIP, estimated Young's Modulus and Poisson's Ratio, and target (lesion) registration error are shown in Table 2. Also we simulate the FEM deformation using the estimated physics-based parameters to perform image fusion as shown in Fig. 5. The CPU time taken is about 5 seconds for online registration, however speed effect of Matlab being an interpreted language should be considered. Through the results, we can observe that, 1) each of the DIP models is compressed significantly compared to the full 1,287 coefficients required for an 8-degree IP; 2) even for complex clinical cases, the deformation occurred in prone-supine images can be accurately simulated using the estimated physics-based parameters.

\section{Discussion and Future Work}

The results of this study suggest that the biomechanical deformation of breast can be represented by a compact DIP, and utilized which a high-speed registration can be achieved for realtime applications. The results demonstrate the potentials for clinical applications such as image-guided breast diagnoses or surgery.

However, currently it still suffers from the difficulty on control of the DIPs, as shown in Table 2, the results of two patients are unsatisfied for the standard accuracy of medical image registration ( $\leq 10 \mathrm{~mm}$ errors at tumors). In future research, we plan to explore several directions: i) Connecting our work to explicit representation models like mutual information in Ref. [7], and to improve the similarity measurement; ii) Studying the techniques of locally controlling the deformation such FFD, and thus to achieve the accurate local registration; iii) Combing more physical cues in FEM to build more strict physical simulation for obtaining training data of deformation.

Acknowledgments The authors express sincere gratitude to Dr. Hiroshi Yoshibayashi at Japanese Red Cross Society Wakayama Medical Center, and the members of the Department of Breast Surgery and Department of Diagnostic Radiology at Kyoto University for their support of this study. This work is partly supported by the Innovative Techno-Hub for Integrated Medical Bio-imaging of the Project for Developing Innovation Systems, from the Ministry of Education, Culture, Sports, Science and Technology (MEXT), Japan.

\section{References}

[1] Blane, M., Lei, Z.B. and Cooper, D.B.: The 3L Algorithm for Fitting Implicit Polynomial Curves and Surfaces to Data, TPAMI, Vol.22, No.3, pp.298-313 (2000).

[2] Carter, T., Tanner, C., Beechey-Newman, N., Barratt, D. and Hawkes, D.: MR Navigated Breast Surgery: Method and Initial Clinical Experi- ence, MICCAI, Vol.LNCS 5242, pp.356-363 (2008).

[3] Guo, Y., Sivaramakrishna, R., Lu, C.-C., Suri, J.S. and Laxminarayan, S.: Breast image registration techniques: A survey, Med. Biol. Eng. Comput., Vol.44, pp.15-26 (2006).

[4] Hu, Y., Morgan, D., Ahmed, H.U., Pendse, D., Sahu, M., Allen, C., Emberton, M., Hawkes, D. and Barratt, D.: A Statistical Motion Model Based on Biomechanical Simulations for Data Fusion during ImageGuided Prostate Interventions, MICCAI, Vol.LNCS 5241, pp.737-744 (2008).

[5] Pathmanathan, P., Gavaghan, D.J., Whiteley, J.P., Chapman, S.J. and Brady, J.M.: Predicting tumor location by modeling the deformation of the breast, IEEE TBE, Vol.55, pp.2471-2480 (2008).

[6] Rajagopal, V., Lee, A., Chung, J.-H., Warren, R., Highnam, R.P. and Nielsen, P.M.F.: Towards Tracking Breast Cancer Across Medical Images Using Subject-specific Biomechanical Models, MICCAI (2007).

[7] Rueckert, D., Sonoda, L.I., Hayes, C., Hill, D.L.G., Leach, M.O. and Hawkes, D.J.: Nonrigid Registration Using Free-Form Deformations: Application to Breast MR Images, IEEE Trans. Medical Imaging, Vol.18, No.8, pp.712-721 (1999).

[8] Zheng, B., Ishikawa, R., Oishi, T., Takamatsu, J. and Ikeuchi, K.: A Fast Registration Method Using IP and Its Application to Ultrasound Image Registration, IPSJ CVA, Vol.9, pp.209-219 (2009).

[9] Zheng, B., Takamatsu, J. and Ikeuchi, K.: An Adaptive and Stable Method for Fitting Implicit Polynomial Curves and Surfaces, TPAMI, Vol.32, No.3, pp.561-568 (2010).

(Communicated by Kensaku Mori) 